\title{
THE
}

\section{Applying Spatial Diversity to Mitigate Partial Band Interference in Undersea Networks}

James McGee

Josko Catipovic

Peter F. Swaszek

University of Rhode Island, swaszek@uri.edu

Follow this and additional works at: https://digitalcommons.uri.edu/ele_facpubs

The University of Rhode Island Faculty have made this article openly available.

Please let us know how Open Access to this research benefits you.

This is a pre-publication author manuscript of the final, published article.

Terms of Use

This article is made available under the terms and conditions applicable towards Open Access Policy Articles, as set forth in our Terms of Use.

\section{Citation/Publisher Attribution}

McGee, J., Catipovic, J., and Swaszek, P., "Applying Spatial Diversity to Mitigate Partial Band Interference in Undersea Networks," Proceedings of the 2014 Wireless Telecommunications Symposium, Washington, D.C., April 2014, pp. 1-8. doi: 10.1109/WTS.2014.6835021

Available at: http://dx.doi.org/10.1109/WTS.2014.6835021

This Conference Proceeding is brought to you for free and open access by the Department of Electrical, Computer, and Biomedical Engineering at DigitalCommons@URI. It has been accepted for inclusion in Department of Electrical, Computer, and Biomedical Engineering Faculty Publications by an authorized administrator of DigitalCommons@URI.For more information, please contact digitalcommons-group@uri.edu. 


\title{
Applying Spatial Diversity to Mitigate Partial Band Interference in Undersea Networks
}

\author{
James McGee and Josko Catipovic \\ Naval Undersea Warfare Center \\ Newport, RI 02841 \\ Email: james.a.mcgee@navy.mil \\ Email: josko.catipovic@navy.mil \\ Peter Swaszek \\ Department of Electrical, Computer and Biomedical Engineering \\ University of Rhode Island \\ Kingston, RI 02881 \\ Email: swaszek@ele.uri.edu
}

\begin{abstract}
Many acoustic channels suffer from interference which is neither narrowband nor impulsive. This relatively long duration partial band interference can be particularly detrimental to system performance. We survey recent work in interference mitigation and orthogonal frequency division multiplexing (OFDM) as background motivation to develop a spatial diversity receiver for use in underwater networks. The network consists of multiple distributed cabled hydrophones that receive data transmitted over a time-varying multipath channel in the presence of partial band interference produced by interfering active sonar signals as well as marine mammal vocalizations. In operational networks, many "dropped" messages are lost due to partial band interference which corrupts different portions of the received signal depending on the relative position of the interferers, information source and receivers due to the slow speed of propagation.
\end{abstract}

\section{Keywords}

Diversity, OFDM, underwater acoustic communications

\section{INTRODUCTION}

W

HILE humans have recognized for millenia that sound can be heard underwater, the ability of humans to communicate underwater over long distances is a recent achievement. Today, a reliable acoustic data link is of great importance for the development of ocean-observation systems and sensor networks. Applications abound, ranging from the oil industry to aquaculture to the military including instrument monitoring, pollution 
control, climate recording, search and survey missions, and the study of marine life [1]. The current vision is for the development of an undersea sensor network, specifically a collection of bottom-mounted instruments and tetherless moving platforms in which different kinds of oceanographic data will be exchanged among the members in real time [2]. Major motivations for the use of wireless communications in the undersea network include 1) reduction in deployment costs and 2) the operational flexibility provided by the ability to monitor and react to sensor information in real or near-real time [1]. Research into the physical layer of undersea networks is extremely active with focus areas including: the improvement of single carrier modulation/detection with more powerful coding techniques and turbo equalization; the exploration of multi-carrier modulation as an alternative to single carrier; and the extension of single-input/single-output (SISO) systems to multi-input/multi-output (MIMO) configurations that provide spatial multiplexing and the ability to send parallel data streams from multiple transmitters [1].

Yet, the deployment of undersea networks is in its infancy. A few short duration experimental networks have been deployed. For example, the Seaweb deployment in 2000 involved 17 nodes spread over $16 \mathrm{~km}^{2}$ for multiple days [3]. The Massachusetts Institute of Technology and Australia's CSIRO (Commonwealth Scientific and Industrial Research Organisation) deployed a network consisting of both fixed and autonomous nodes in very near-shore areas of Australia [4].

To date the only long term undersea cellular network is operated by the U. S. Navy in the Tongue of the Ocean [5]. Known as the Atlantic Undersea Test and Evaluation Center (AUTEC), it consists of 96 acoustic sensors placed over a 60 by 30 kilometer square area and is shown in Fig. 1. As currently configured, approximately 97 percent of transmitted messages are successfully decoded; of the remaining three percent, many are corrupted by acoustic interference arising from active acoustic emissions. For example, Fig. 2 shows the impact of interference on a received data packet. In pane (a), the data packet was received without interference and successfully decoded in contrast to pane (b) where interfering signals are clearly evident and the message was lost. Years of extensive observations of activities in the vicinity of the network demonstrate that the widely separated hydrophones suffer from partial-band interference emanating from multiple spatially separated sources. The nature of this interference is different from the impulsive or narrowband interference typically encountered in other applications. Furthermore, unlike RF communications and acoustic array processing applications where interference is highly correlated in time among the various receivers, in the acoustic network, interference affects different portions of the received signals due to the wide separation of the receivers and the low speed of propagation. The degradation in the received signal is highly variable, depending on the relative position of the interfering signals, information source and receivers as well as the channel conditions. While successful steps to mitigate interference have recently been reported [6], utilizing the spatial diversity implicit in the undersea network to mitigate interference has not yet been attempted. The motivation of this work is to examine the potential benefits that leveraging spatial diversity in underwater acoustic networks might provide.

\section{A. Challenges of the Underwater Channel}

While the spatial diversity implicit in the network offers the opportunity to mitigate interference, the diversity receiver must address the challenges the underwater acoustic channel presents, namely: 1) severely limited operational bandwidth, 2) significant multipath spread, 3) non-stationary statistics, and 4) the need for explicit time synchronization in mobile applications (see e.g. [7]-[10]). As is the case at AUTEC, many acoustic channels 


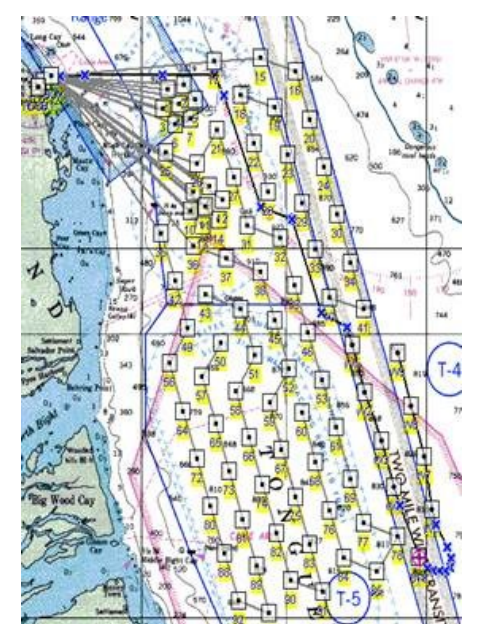

Figure 1. The AUTEC acoustic network.

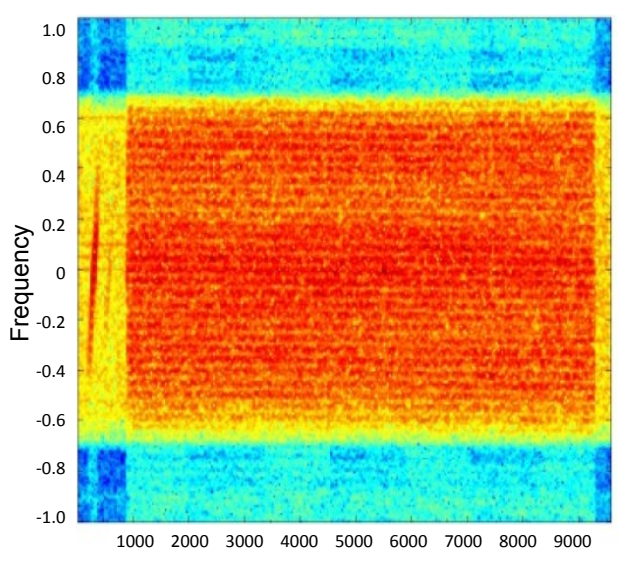

Figure 2. Clean packet reception (a) and packet corrupted by acoustic interference (b).

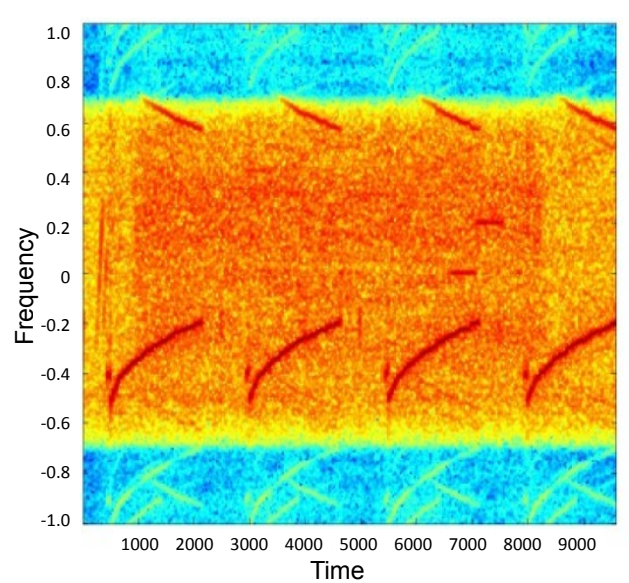

(b)

suffer from interference which is neither narrowband nor impulsive [6]. This relatively long duration partial band interference can be particularly detrimental to communication systems that do not account for it. While the challenges the underwater channel presents are manifold, the diversity inherent in the channel offers opportunities to ameliorate them.

Diversity techniques are well known to improve the reliability of communications over fading multipath channels and rely on the notion that errors occur in reception when the channel attenuation is large; that is, when the channel is in a deep fade [11]. Diversity techniques may be classified as either explicit, where the message is intentionally transmitted through different uncorrelated parts of the signal space, or implicit, where the message occupies the entire signal spectrum and the receiver tries to identify it by compensating for the channel distortion [12]. The central idea behind both approaches is to supply the receiver with multiple replicas of the same information transmitted over independently fading portions of the channel, thereby reducing the probability that all signal components will 
fade simultaneously.

Diversity techniques can be employed on selective channels, where selectivity refers to the differential enhancement or attenuation exhibited in the received signal resulting from transmission through the channel with respect to a particular parameter. For example, a frequency selective channel attenuates different frequency bands of the transmitted signal differently, and its frequency response varies over its bandwidth. The underwater acoustic channel exhibits time, frequency and spatial selectivity as a consequence of the physics of sound propagation in the ocean. Frequency selectivity arises from the absorption losses which increase with signal frequency. The coherence bandwidth of the channel, the range of frequencies over which the channel gain is almost constant, is on the order of $10 \mathrm{Hertz}$ at a transmit frequency of $10 \mathrm{kHz}$. Time and spatial selectivity result from time-varying multipath propagation, and the low speed of sound $(1500 \mathrm{~m} / \mathrm{s})$. Multipath formation is governed by sound reflection and refraction. Reflection occurs at the surface and bottom and off any objects in the medium. Refraction results from the spatial variability of sound speed which depends on the temperature, salinity, and pressure. The multipath delay spread, the time delay between the first and last significant arrival of a transmitted signal, can range from 10 milliseconds in shallow water at short ranges to hundreds of milliseconds in deep ocean channels. The multipath structure is highly environmentally dependent and the impulse response may be sparse exhibiting a few large amplitude taps separated by many taps of small magnitude.

Changes in the propagation medium and transmitter/receiver motion induce time variability in the channel. Changes in the propagation medium occur over different time scales ranging from seasonal temperature changes to much faster fluctuations that affect the signal such as surface wave action. Wave motion causes scattering of the signal, and rapid fluctuations in signal path length induces Doppler spreading. The slow speed of sound relative to the speed of mobile transmitters results in significant variability to the channel through the Doppler effect, which causes frequency shifting as well as frequency spreading. The resulting channel exhibits significant time variability. Coherence time, a measure of the time scale over which the channel changes "significantly", can range from seconds in extremely stable situations to 100 milliseconds in rapidly fluctuating channels.

\section{B. Survey of Recent Work}

While acoustic communications have historically used a single carrier frequency, within the last decade research has focused on multi-carrier modulation and OFDM in particular, (see [13]-[16] and references therein). OFDM divides the doubly (time- and frequency-) selective underwater channel into multiple orthogonal subbands. The bandwidth of each subband is chosen to be less than the coherence bandwidth of the channel so that each subband experiences relatively flat fading, thereby simplifying the channel equalization problem. Additionally, Inter-Symbol Interference (ISI) is mitigated since the transmission scheme may be viewed as transmitting many parallel low-rate narrowband signals rather than one fast-rate wideband signal [31]. The effects of ISI may be further reduced by inserting a guard interval between OFDM symbols that is filled with either a cyclic prefix (CP-OFDM) or a zero prefix (ZP-OFDM). Trade-offs between the two prefix approaches are discussed in [17].

Although OFDM mitigates ISI, inter-carrier interference (ICI) resulting from Doppler induced shifting of signals from one subband into another can severely degrade performance. Motion-induced Doppler distortion presents a major obstacle in applying OFDM to underwater channels since both the channel impulse response and the nonuniform effects of relative motion on subbands must be estimated. Solutions to this problem are found in two 
broad approaches [16]: 1) adaptive synchronization, which requires few subbands dedicated to channel estimation but relies on coherence between adjacent OFDM blocks, and 2) non-adaptive synchronization, which does not rely on channel coherence but requires null subcarriers to gain robustness to fast channel variations. Selection and implementation of an appropriate technique depends on the coherence time and coherence bandwidth of the channel as well as making appropriate tradeoffs among computational complexity, required channel estimation accuracy, and data rate. Distributing pilot tones evenly throughout the band and performing interpolation is more efficient than periodically dedicating an entire OFDM symbol to channel estimation [18]. Iterating the equalization and decoding tasks, that is performing "turbo equalization", can yield large performance improvements [19], and reduced-complexity approximate algorithms are available as in [20] for example.

Through appropriate Doppler compensation of the received signal and judicious assignment of codewords to data slots within an OFDM packet, explicit time and frequency diversity can be achieved. Assigning parts of a codeword to subbands separated by more than the coherence bandwidth of the channel achieves frequency diversity, while using an interleaving depth greater than the coherence time of the channel provides time diversity. Experimental results with OFDM have achieved data rates on the order of tens of kilobits per second but none of the algorithms have attempted to account for interference while taking advantage of spatial diversity. The algorithms in [6] and [13] address the possibility of interference but do not provide spatial diversity, while the systems developed in [12] achieve time, frequency, and spatial diversity for a vertical line array but do not address interference. The asynchronous multiuser OFDM algorithm proposed in [21] views interfering users as a single external interference, which the algorithm parameterizes and uses in an iterative channel estimation, data detection and interference mitigation scheme on a single receiver. Observations from past field experiments indicate that significant improvement in the reliability of message reception can be realized by mitigating interference. Discussion of the interference typical in the underwater environment is available in [22] and [23] .

Interference mitigation has a long history in RF communications but the interference is typically impulsive or narrowband [24]. Partial band interference is not addressed [6]. The interference mitigation techniques typically exploit the short time or limited frequency span of the interfering signal. Examples of impulsive noise suppression techniques for multi-carrier modulation may be found in [25]-[31], while [32]-[41] address narrowband interference mitigation. Early approaches tended to separate channel estimation and interference detection, and more recent work has focused on jointly estimating the channel and mitigating interference. Joint approaches may work iteratively such as in [26] or by expanding the states of the decoding algorithm as in [36] and [38]. A message-passing approach to jointly estimating the channel and mitigating strong co-channel interference of similar form as the desired signal was proposed in [42]. Two blind algorithms to mitigate multiple interferers were proposed in [43]. Joint approaches provide better performance at the cost of additional computational complexity. Limitations on system performance may be found in [44] for OFDM systems subject to impulsive noise and for multicarrier and single carrier quadrature amplitude modulation (QAM) systems in [45]. The capability of low density parity check (LDPC) and turbo coding to mitigate burst errors is discussed in [46].

In mobile radio, optimal receiver combining [47] has been used for years to combat interference and the performance bounds were developed in [48]; but, the channel is much less complicated than in underwater communications: it contains no multipath and Doppler effects may be safely ignored. A receiver that addressed the challenges of the underwater channel and performed joint diversity combining of multiple channel receptions with channel equalization 
was proposed in [49]. More recently, [50] proposed a multiple-input multiple output system which used space-time coding and iterative decoding techniques to obtain high data rates over shallow-water, medium range channels in the absence of interference. In the 200-300 Hz frequency range, [51] exploited beam diversity among sensors separated by hundreds of meters to improve communication performance.

Whereas processing time and computational complexity are critical drivers in many RF applications (such as mobile phones and digital audio and video broadcasting), underwater communication applications exist (such as submarine to shore message trafficking) where decoding delays are tolerable and vast amounts of computational power are available. Thus, the additional available decoding time and computing power may be employed to untangle the more complicated channel and Doppler effects.

To recapitulate, the motivation for this research is to examine approaches to leverage the spatial diversity of underwater acoustic communications networks suffering from interference which degrades different portions of the received signal depending on the source-receiver-interferer geometry.

\section{Spatial Diversity At AUTEC}

Acoustic propagation is much more environmentally variable than RF, largely due to the slow $\sim 1500 \mathrm{~m} / \mathrm{sec} \mathrm{speed}$ of propagation and its strong dependence on ocean temperature, pressure and salinity. Fig. 3 illustrates this variability from a networking point of view: thirty-nine messages were transmitted from the location marked by the blue dot, numbers of receptions at receiver hydrophones are indicated by the number adjacent to each phone, and the relative signal strength and quality, as assessed by the AUTEC ACOMMs processing algorithm, is represented by color. As expected, receivers close to the transmitter receive the strongest signals, although even in this case the number of high-quality receptions drops off precipitously with range: a receiver less than four miles away only received seven high-quality receptions. The red-marked receivers indicate correctly-decodable receptions with degraded SNR and/or enhanced or dynamic multipath. The black receivers did not receive any decodable transmissions, although their ranges to the transmitter are in many cases comparable to hydrophones with excellent reception. This patterning is very typical of the oceanic area and arises from spatial fluctuations of three-dimensional temperature distribution in the ocean. Correlation of reception intensity or quality between successive packet transmissions is typically low.

\section{A SPATIAl DiVERsity ReCEIVER}

The work in [6] developed the single receiver algorithm consisting of the grey boxes shown in Fig. 4 and performed the following functions:

- Doppler Compensation is performed individually for each received signal. It compensates for the effects of relative motion between the source and receiver.

- Interference Detection determines whether interference is present.

- Interference Subtraction removes interference from the received signal, if present.

- Channel Estimation is based on pilot tones and soft decisions fed back from the multi-receiver LDPC decoding algorithm.

- Noise Variance Update estimates the noise level to enable appropriate weighting of the received measurements. 


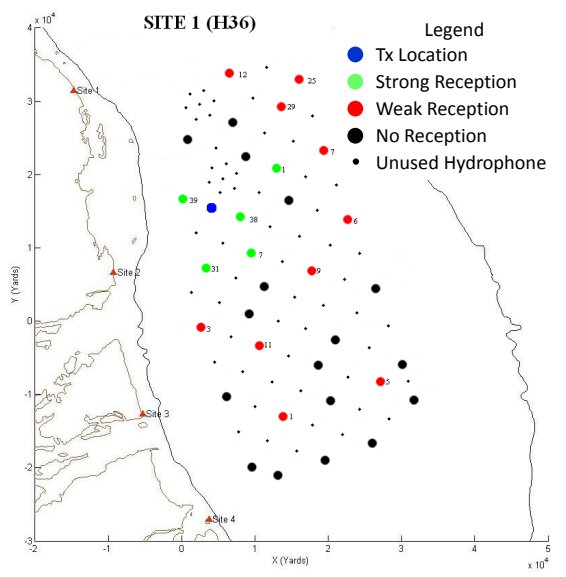

Figure 3. Receptions as a function of geometry from the transmitter. Larger circles indicate the thirty-two hydrophones used for communication signal reception.

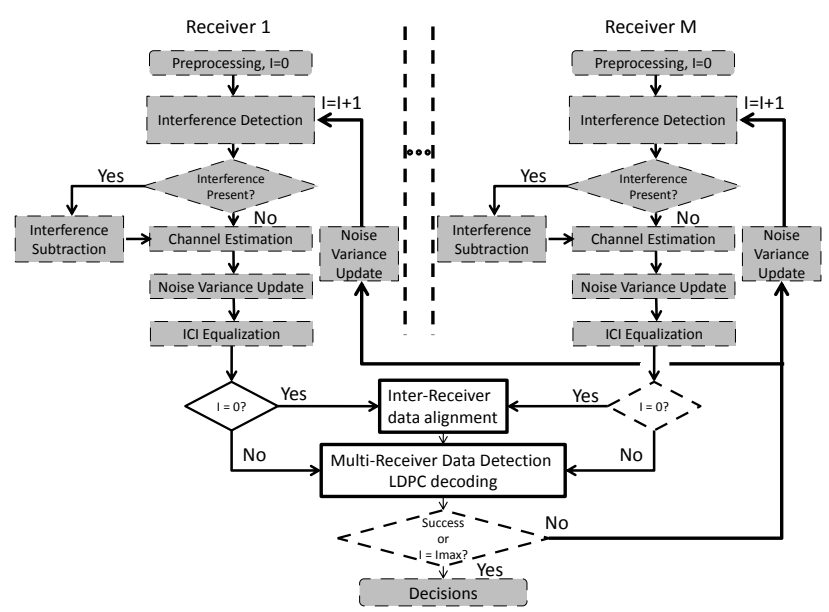

Figure 4. Spatial diversity receiver.

- Channel Equalization is performed using a standard technique such as zero-forcing, or minimum mean square error (MMSE). The output of the equalization process is a measurement for each data symbol for each receiver which the decoding algorithm uses as extrinsic information.

Building upon the work in [6], we seek to develop a spatial receiver which takes advantage of the geographical separation of the hydrophones resulting in the interference arriving at different times and lasting for different durations in the received signal. It performs the following additional functions which are shown in white boxes in figure 5:

- Inter-receiver Time Alignment aligns the received measurement information.

- Multi-receiver data detection and LDPC decoding. The received waveform is reconstructed from the clean portions of the received signals which suffered from time and band limited interference. Data detection is 


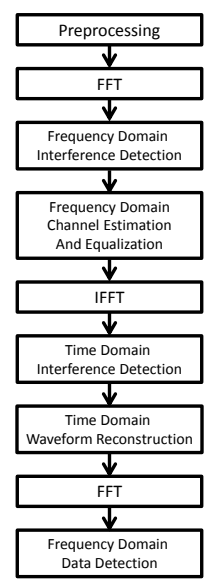

Figure 5. OFDM waveform reconstruction process.

performed on the reconstructed waveform followed by LDPC decoding. In the event that decoding fails, successfully decoded symbols are provided as input to the channel estimation and channel equalization algorithms on the next iteration.

The waveform reconstruction process is illustrated in Fig. 5. The interference is time and band limited, and as in [6], we assume these parameters are known. The reconstruction process identifies the portions of the received waveforms suffering from interference and then optimally combines the remaining clean portions of the signal. The reconstruction process must occur in the time domain since the interference occupies the same frequency band on all receivers but arrives at different times on different receivers. Furthermore, it is essential that the reconstruction process operate on equalized waveforms. All of the information for channel estimation and residual Doppler compensation is present in the frequency (or OFDM symbol) domain. Consequently, after signal detection and gross Doppler compensation, the reconstruction process begins with interference detection/suppression in the frequency domain followed by channel equalization. The algorithms developed in [6] and the references therein can be used for these tasks. The equalized received signals must then be transferred back to the time domain to remove the residual time orthogonal interference. The time domain interference detector may take advantage of information gained from the frequency domain interference detector. Portions of the received signals where interference is declared are excised, provided a clean copy of the same portion of the waveform exists on another receiver. The synthesized signal is then transferred back to the frequency domain for data detection.

To demonstrate the concept of waveform reconstruction, we implemented a simple frequency domain interference detector based on comparing the energy in the null subcarriers in the interference band to energy in the null subcarriers in the noise only band. If the frequency domain interference detector declared interference present, the time domain interference detector selected an appropriately sized contiguous window with the highest signal energy for potential excision. A brief description of the two detectors and the reconstruction procedure follows.

The zero-padded OFDM signal consists of $K$ subcarriers which are divided into non-overlapping sets of active subcarriers $\mathcal{S}_{A}$ and null subcarriers $\mathcal{S}_{N}$ satisfying $\mathcal{S}_{A} \cup \mathcal{S}_{N}=\{-K / 2 \ldots K / 2-1\}$. The transmitted time domain 
symbol $s[n]$ is related to the OFDM data symbol through the inverse Fourier transform. Specifically,

$$
s[n]=\sum_{k=-K / 2}^{K / 2-1} d[k] e^{j 2 \pi \frac{k n}{K}}
$$

so that $\mathbf{s}=\operatorname{IFFT}(\mathbf{d}, K)$. The input-output relationship between the transmitted symbols, $d[m]$, and the discrete frequency sample $z[k]$ may be written as

$$
z[k]=\sum_{m=-K / 2}^{K / 2-1} \mathbf{H}[k, m] d[m]+w[k]+v[k],
$$

where $\mathbf{H}$ describes the frequency response of the channel, $w$ is additive noise assumed to be white Gaussian noise and $v$ is the interference.

Let $\mathcal{S}_{w}$ denote the subcarriers in the noise only band, and $\mathcal{S}_{v}$ denote the subcarriers in the band which potentially suffers from interference. The frequency domain interference detector declares interference if

$$
\frac{1}{\left|\mathcal{S}_{v}\right|} \sum_{k \in \mathcal{S}_{v}}|z[k]|^{2}>\frac{1}{\left|\mathcal{S}_{w}\right|} \sum_{k \in \mathcal{S}_{w}}|z[k]|^{2}
$$

and the Komogorov-Smirnov hypothesis test determines the samples $z\left[k \epsilon \mathcal{S}_{v}\right]$ and $z\left[k \epsilon \mathcal{S}_{w}\right]$ to be from different distributions with significance level of greater than five percent. The MATLAB® function kstest 2 may be used to perform the hypothesis test.

Provided interference is detected, the received frequency samples are transformed to the time domain, $\mathbf{x}=$ $\operatorname{IFFT}(\mathbf{z}, K)$. A rolling window of size $L$ sums the energy in the time domain signals. For this work, we chose $L$ so that the window was $1.05 T_{I}$. Because the frequency domain samples in ZP-OFDM are formed from overlapping and adding samples from the guard period with samples in the symbol period, the window "wraps" around $\mathrm{x}$, that is

$$
y[k]=\sum_{n=0}^{L-1}|x[\bmod (k+n, K)]|^{2}
$$

Time domain interference is declared in the window $i=\bmod \left(k_{\max }: k_{\max +L-1}, K\right)$ where $k_{\max }$ denotes the index where $y$ achieves its maximum. Let $I_{k, r}$ denote the indicator function of interference in the $k$ th band on the $r$ th receiver, and similarly concatenate the received signals into a matrix $\mathbf{Z}_{k, r}$. The signal reconstruction operation on the $R$ receivers is then defined by

$$
\overline{\mathbf{z}}_{k}=\left\{\begin{aligned}
\frac{\sum_{r} \mathbf{Z}_{k, r} \circ \mathbf{I}_{k, r}}{\sum_{r} \mathbf{I}_{k, r}} & \text { where } \sum_{r} \mathbf{I}_{k}>0 \\
\frac{\sum_{r} \mathbf{Z}_{k, r}}{R} & \text { where } \sum_{r} \mathbf{I}_{k}=0
\end{aligned}\right.
$$

where $\circ$ denotes the Hadamard (element-wise) matrix product. Thus, non-contemporaneous interference is excised from the reconstructed signal while averaging is performed across all portions of the signals where the interference occurs concurrently on all receivers resulting in a clean portion of the signal being unavailable.

\section{Simulation}

The waveform reconstruction algorithm was tested using simulated data which assumed the geometry shown in Fig. 6. The source is equidistant from the receivers and the interfering source is closer to receiver 1 than receiver 


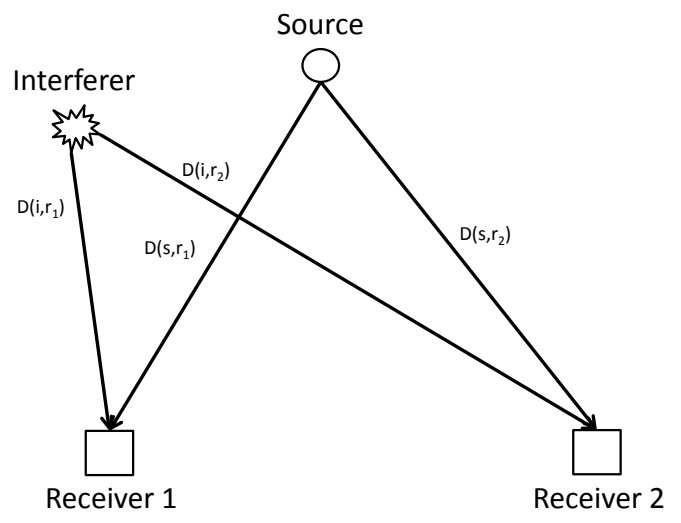

Figure 6. Simulation geometry. $D\left(s, r_{1}\right)=D\left(s, r_{2}\right)$ and $D\left(i, r_{1}\right)<D\left(i, r_{2}\right)$.

2. The simulated OFDM signal and interference parameters match those used in the work of [6] but the channels, which are shown in Fig. 7, are much more benign than the ten tap Rayleigh channels simulated in [6]. We assume both channels are known and equalize the receptions by inverting the channel response; that is, we employ a zeroforcing equalizer. On channel 1, which is taken from [11] figure 9.4-5, the interference arrives in the first half of the OFDM symbol period whereas the interference corrupts the second half of the received signal on channel 2. Table IV lists the simulation parameters.

The interference is generated by passing white Gaussian noise of time duration $T_{I}=T / 4$ ms through a bandpass filter with a center frequency of $15 \mathrm{kHz}$ and bandwidth of $2.4 \mathrm{kHz}$. The delay of the interference relative to the start of each block is uniformly distributed according to the start time parameter listed in table IV. The interference is thus orthogonal in the time domain on the two receivers but overlaps in the frequency domain.

The simulated time domain interference is sampled, overlapped and added, and a $K$ point FFT is taken to produce frequency domain interference which is then scaled to the appropriate signal-to-interference (SIR) level and added to the background noise, which is modeled as complex white Gaussian noise with signal-to-noise ratio (SNR) of $7.9 \mathrm{~dB}$. The SIR and SNR are defined as

$$
S I R=\frac{P_{s}}{P_{I}} \quad S N R=\frac{P_{s}}{N_{0}}
$$

where $P_{s}$ is the average power of the OFDM frequency measurements within the useful signal band, $P_{I}$ is the average power of the interference frequency components within the interference frequency band, and $N_{0}$ is the variance of the additive noise in the frequency domain. After adding the simulated noise to an OFDM symbol vector, the waveform reconstruction algorithm was run at a SNR of $7.9 \mathrm{~dB}$ for SIRs varying from -10 to $2 \mathrm{~dB}$. The Monte Carlo simulation was stopped when either 500,000 bits had been processed or 250 errors were made. 

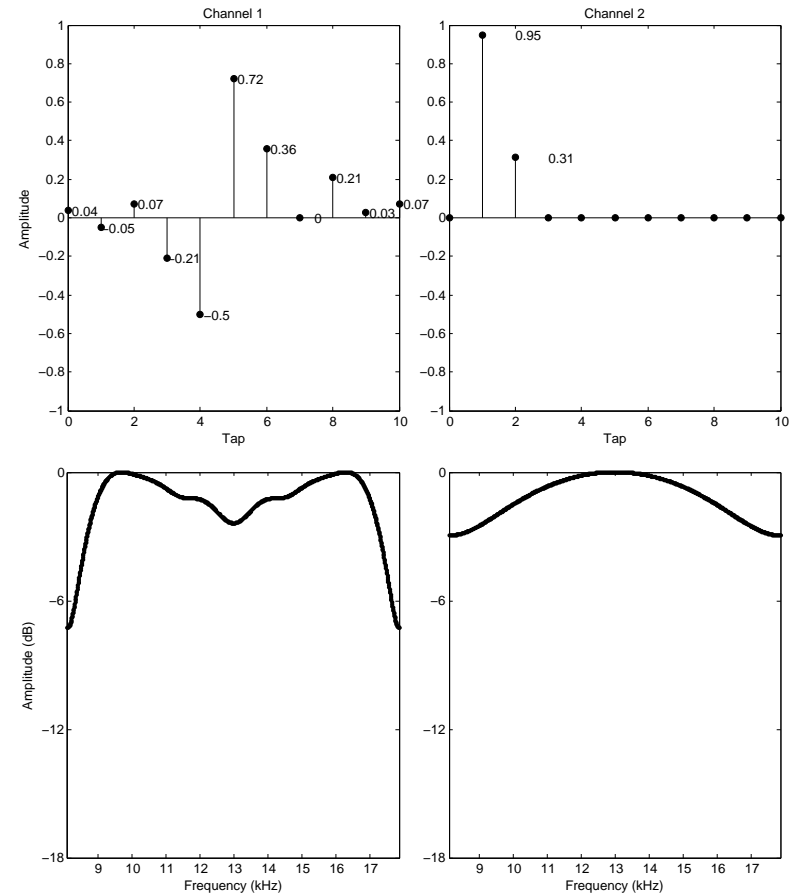

Figure 7. Simulated Channels.

\begin{tabular}{|l|l|l|}
\hline \multicolumn{2}{|c|}{ OFDM Parameter } & Value \\
\hline Center frequency & $f_{c}$ & $13 \mathrm{kHz}$ \\
\hline Bandwidth & $B$ & $9.77 \mathrm{KHz}$ \\
\hline \# of subcarriers & $K$ & 1024 \\
\hline \# data subcarriers & $\left|\mathcal{S}_{D}\right|$ & 672 \\
\hline \# pilot subcarriers & $\left|\mathcal{S}_{P}\right|$ & 256 \\
\hline \# null subcarriers & $\left|\mathcal{S}_{N}\right|$ & 96 \\
\hline Symbol Duration & $T$ & $104.68 \mathrm{~ms}$ \\
\hline Symbol Constellation & & BPSK \\
\hline Subcarrier spacing & $\Delta f=1 / \mathrm{T}$ & $9.54 \mathrm{~Hz}$ \\
\hline Guard interval & $T_{g}$ & $24.6 \mathrm{~ms}$ \\
\hline Number of Guard Samples & $N_{g}$ & 240 \\
\hline \multicolumn{2}{|c|}{ Interference Parameter } & Value \\
\hline Center Frequency & $f_{c, I}$ & $15 \mathrm{kHz}$ \\
\hline Bandwidth & $B_{I}$ & $2.4 \mathrm{kHz}$ \\
\hline Duration & $T_{I}$ & $26.2 \mathrm{~ms}$ \\
\hline Channel 1 Start time & $T_{s, 1}$ & $\mathcal{U}\left(.1 T_{I}, T / 2-1.1 T_{I}\right)$ \\
\hline Channel 2 Start time & $T_{s, 2}$ & $\mathcal{U}\left(T / 2+.1 T_{I}, T-1.1 T_{I}\right)$ \\
\hline
\end{tabular}

Table I. SimUlation PARAMETERS. 


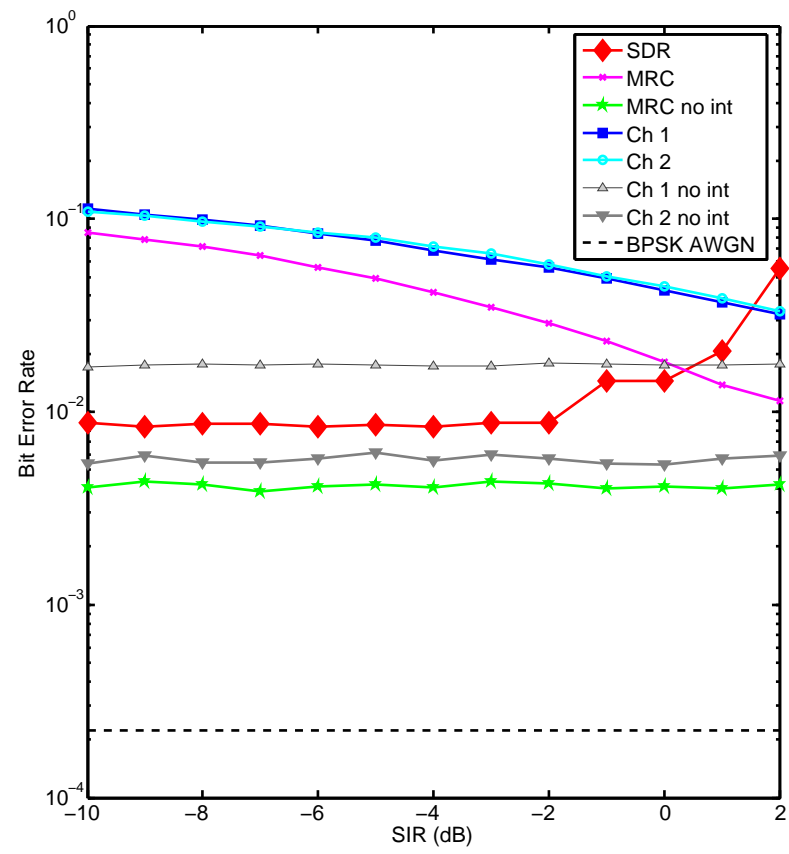

Figure 8. Bit Error Rate for different combining strategies at an SNR of $7.9 \mathrm{~dB}$ and various SIRs: SDR - Spatial Diversity Reconstruction, MRC - Maximum Ratio Combining, Ch1,Ch2 - single channel, No Int - No interference present, BPSK AWGN - binary phase shift keying on an impulse channel in additive white Gaussian Noise.

\section{RESUlts}

Fig. 8 clearly demonstrates the benefits of leveraging spatial diversity to reconstruct the transmitted waveform. Spatial diversity reconstruction (SDR) performance is better than the single receiver performance on channel 1 with no interference and slightly worse than the interference-free performance for a single receiver on channel 2 . This is expected since the impulse response of channel 1 causes more intersymbol interference than the impulse response of channel 2. The performance of the implemented detectors decreases as the SIR improves above $-1 \mathrm{~dB}$ because the interference becomes more difficult to detect. In this SIR regime, the detector mistakenly excises portions of the signal that do not contain interference. The single receiver performance of binary phase shift keying on an additive white Gaussian noise channel with no interference is shown for comparison. Channel equalization plays a critical role in performance not only because better equalization improves the averaging operation in the time domain, but critically because any noise enhancement resulting from equalization is smeared across the time series through the subsequent Fourier transform operation. Thus, a minimum mean square equalizer would probably provide better performance than the zero forcing equalizer employed here. 


\section{SUMMARY}

Many acoustic channels suffer from interference which is neither narrowband nor impulsive. This relatively long duration partial band interference can be particularly detrimental to system performance. Surveying the challenges of the acoustic channel and reviewing the recent work in interference mitigation and OFDM provides motivation for the development of a spatial diversity receiver. Operating simple detectors on relatively benign channels, we demonstrated that leveraging spatial diversity to reconstruct the transmitted waveform results in significant performance improvement over the classical maximum ratio combining strategy at high signal-to-interference ratios.

\section{REFERENCES}

[1] J. Heidermann, M. Stojanovic, , and M. Zorzi, “Underwater sensor networks: Applications, advances, and challenge,” Royal Society, 2012.

[2] D. Green, "Acoustic modems, navigation aids, and networks for undersea operations," in Proc. IEEE Oceans Conference, Marco Island, FL, Jan. 2010.

[3] J. Proakis, E. Soxer, J. Rice, and M. Stojanovic, "Shallow water acoustic networks," IEEE Communications Magazine, vol. 39, no. 11, pp. 114-119, 2002.

[4] I. Vasilescu, R. Kotay, D. Rus, M. Dunbabin, and P. Corke, "Data collection, storage, and retrieval with an underwater sensor network," in Proc. of the third ACM SenSys conference, no. 3, San Diego, June 2005, pp. 154-165.

[5] R. Hayford, D. Nagle, and J. Catipovic, "Undersea cellular network, tongue of the ocean bahamas," in Proc. of the 4th ACM Workshop on Underwater Network (WuWNet), Berkerly, 2009.

[6] Z. Wang, S. Zhou, J. Catipovic, and P. Willett, "Parameterized cancellation of partial-band partial-block-duration interference for underwater acoustic OFDM," IEEE Transactions on Signal Processing, vol. 60, no. 4, pp. 1782-1795, 2012.

[7] R. J. Vacarro, "The past, present and future of underwater acoustic signal processing," IEEE Signal Processing Magazine, vol. 15, no. 4, pp. 21-51, 1998.

[8] M. Stojanovic, "Underwater Acoustic Communication" in Encyclopedia of Electrical and Electronics Engineering. Wiley, 2000.

[9] J. A. Catipovic, "Performance limitations in underwater acoustic telemetry," IEEE Journal of Oceanic Engineering, vol. 15, no. 3, pp. 205-216, 1990.

[10] M. Stojanovic and J. Presig, "Underwater communication channels: Propagation models and statistical characterization," IEEE Communications Magazine, pp. 84-89, January 2009.

[11] J. Proakis and S. Masoud, Digital Communications, 5th ed. McGraw-Hill, 2008.

[12] K. Pelekanakis, "Harvesting time-frequency-space diversity with coded modulation for underwater acoustic communications," Ph.D. dissertation, Massachusetts Institute of Technology, 2009.

[13] H. Sun, W. Shen, Z. Wang, S. Zhou, X. Xu, and Y. Chen, "Joint carrier frequency offset and impulse noise estimation for underwater acoustic OFDM with null subcarriers," in Proc. IEEE Oceans Conference, 2012.

[14] B. C. Kim and I. T. Lu, "Parameter study of OFDM underwater communications system," in Proc. MTS/IEEE Oceans, Providence, RI, 2000.

[15] J. Z. Huang, S. Zhou, J. Huang, C. R. Berger, and P. Willett, "Progressive inter-carrier interference equalization for OFDM transmission over time-varying underwater acoustic channels," in Proc. MTS/IEEE Oceans Conference, 2010.

[16] M. Stojanovic, "OFDM for underwater acoustic communications: Adaptive synchronization and sparse channel estimation," ICASSIP, p. 5288, 2008.

[17] B. Muquet, Z. Wang, and G. Giannakis, "Cyclic prefix or zeropadding for wireless multicarrier transmissions?" IEEE Trans. Commun., vol. 50, no. 12, pp. 2136-2148, Dec. 2002.

[18] R. Negi and J. Cioffi, "Pilot tone selection for channel estimation in a mobile OFDM system," IEEE Trans. Consumer Electronics, vol. 44, no. 3, Aug 1998. 
[19] M. Tuchler, R. Koetter, and A. C. Singer, "Turbo Equalization: Principles and New Results," IEEE Trans. Commun., vol. 50, no. 5, pp. 754-766, 2002.

[20] A. C. S. M. Tuchler and R. Koetter, "Minimum mean squared error equalization using a priori information," IEEE Trans. Signal Process., vol. 50, no. 3, pp. 673-683, 2002.

[21] J. C. Z. Wang, Z. Shengli and P. Willett, "Asynchronous multiuser reception for OFDM in underwater acoustic communications," IEEE Transactions on Wireless Communications., vol. 12, no. 3, pp. 1050-1061, Mar. 2013.

[22] J. Ward, M. Fitzpatrick, N. Dimarzio, D. Moretti, and R. Morrizzey, "New algorithm for open ocean mammal monitoring," in Proc. MTS/IEEE OCEANS Conf., 2000.

[23] J. Catipovic, "Robust acoustic communication with underwater vehicles in noisy and jammed shallow water environments," in OCEANS 1992: Mastering the Oceans Through Technology, 1992.

[24] J. Laster and J. Reed, "Interference rejection in digital wireless communications," IEEE Signal Process. Mag., vol. 14, no. 3, pp. 37-62, 1997.

[25] S. Zhidkov, "Impulsive noise suppression in OFDM-based communication systems," IEEE Trans. Consum. Electron., vol. 49, no. 4, pp. 944-948, 2003.

[26] A. Mengi and A. Vinck, "Successive impulsive noise suppression in OFDM," in Proc. IEEE Int. Symp. on Power Line Commun. Appl., 2010.

[27] M. F.-G. Garc;a, J. Rojo-Alvarez, F. Alonso-Atienza, and M. Martinez-Ramøn, "Support vector machines for robust channel estimation in OFDM,” IEEE Signal Process. Lett., vol. 13, no. 7, pp. 397-400, 2006.

[28] X. Yu, P. Lin, Z. He, and W. Wu, "OFDM channel estimation with impulse noise cancellation," in Proc. Int. Conf. on Wireless Commun., 2007, pp. 330-333.

[29] G. Caire, T. Al-Naffouri, and A. Narayanan, "Impulse noise cancellation in OFDM: An application of compressed sensing," in Proc. IEEE Int. Symp. Inf. Theory, 2008, pp. 1293-1297.

[30] K. Pelekanakis, H. Liu, and M. Chitre, "An algorithm for sparse underwater acoustic channel identification under symmetric alpha-stable noise," in Proc. of MTS/IEEE OCEANS Conf., 2011.

[31] J. Armstrong and H. A. Suraweera, "Decision directed impulse noise mitigation for OFDM in frequency selective fading channels," in Proc. GLOBALCOM, 2004, pp. 3536-3540.

[32] R. Nilsson, F. Sjoberg, and J. LeBlanc, "A rank-reduced lmmse canceller for narrowband interference suppression in OFDM-based system," IEEE Trans. Commun., vol. 51, no. 12, pp. 2126-2140, 2003.

[33] D. Darsena, "Successive narrowband interference cancellation for OFDM systems," IEEE Commun. Lett., vol. 11, pp. 73-75, 2007.

[34] D. Darsena and F. Verde, "Successive NBI cancellation using soft decisions for OFDM systems," IEEE Signal Process. Lett., vol. 15, pp. 873-876, 2008.

[35] A. Jeremic, T. A. Thomas, and A. Nehorai, "OFDM channel estimation in the presence of interference," IEEE Trans. Signal Process., vol. 52, no. 12, pp. 3429-3439, 2004.

[36] M. Morelli and M. Moretti, "Channel estimation in OFDM systems with unknown interference," IEEE Trans. Wireless Commun., vol. 8, no. 10 , pp. $5338-5347,2009$.

[37] M. Morelli and M. Morett, "Improved decoding of BICM-OFDM transmissions plagued by narrowband interference," IEEE Trans. Wireless Commun., vol. 10, no. 1, pp. 20-26, 2011.

[38] T. Li, W. H. Mow, V. Lau, M. Siu, R. Cheng, and R. Murch, "Robust joint interference detection and decoding for OFDM-based cognitive radio systems with unknown interference," IEEE J. Sel. Areas Commun., vol. 25, no. 3, pp. 566-575, 2007.

[39] A. Gomaa, K. M. Z. Islam, and N. Al-Dhahir, "Two novel compressive-sensing algorithms for NBI detection in OFDM systems," in Proc. Int. Conf. on Acoust., 2010, pp. 3294-3297.

[40] T. Al-Naffouri, A. Quadeer, and G. Caire, "Impulsive noise estimation and cancellation in dsl using orthogonal clustering," in Proc. IEEE Int. Symp. Inf. Theory, 2011, pp. 2841-2845. 
[41] K. Fazel, "Narrow-band interference rejection in orthogonal multicarrier spread-spectrum communications," in Third Annual International Conference on Universal Personal Communications, 1994, pp. 46-50.

[42] Y. Zhu, D. Guo, and M. L. Honig, "A message-passing approach for joint channel estimation, interference mitigation, and decoding," IEEE Trans. Wireless Commun., vol. 8, no. 12, pp. 6008-6018, 2009.

[43] F. He, X. Wang, T. Yang, B. Hu, and R. Liu, "Two blind algorithms of mmse filter based on second-order statistics in mimo systems with multiple interferences," in Wireless Communications and Signal Processing (WCSP), 2011.

[44] S. V. Zhidkov, "Performance analysis and optimization of OFDM receiver with blanking nonlinearity in impulsive noise environment," IEEE Trans. Vehicular Technology, vol. 55, no. 1, pp. 234-242, Jan. 2006Jan.

[45] M. Ghosh, "Analysis of the effect of impulse noise on multicarrier and single carrier QAM systems," IEEE Trans. Commun., vol. 44, no. 2, pp. 145-147, 1996.

[46] S. J. Johnson, Iterative Error Correction: Turbo, Low Density Parity-Check and Repeat Accumulate Codes. New South Wales: Cambridge University Press, 2010.

[47] J. H. Winters, "Optimum combining in digital mobile radio with cochannel interference," IEEE Journal on Sel. Areas in Communications, no. 2, pp. 528-539, 1984.

[48] M. Chiani, M. Z. Win, A. Zanella, R. K. Mallik, and J. H. Winters, "Bounds and approximations for optimum combining of signals in the presence of multiple cochannel interferers and thermal noise," IEEE Transactions on Communications, vol. 51, no. 2, pp. 296-306, Feb. 2003Feb.

[49] M. Stojanovic, J. A. Catipovic, and J. G. Proakis, "Adaptive multichannel combining and equalization for underwater acoustic communications," J. Acoust. Soc. Amer., vol. 94, no. 3, pp. 1621-1631, 1993.

[50] S. Roy, T. M. Duman, V. McDonald, and J. G. Proakis, "High rate communication for underwater acoustic channels using multiple transmitters and space-time coding: Receiver structures and experimental results," IEEE J. Ocean. Eng., vol. 32, no. 3, pp. 663-688, Jul. 2007.

[51] H.-C. Song, "Long-range acoustic communication in deep water using a towed array: Beam diversity," in OCEANS, Yeosu 2012. 